\title{
La influencia de un recurso informático en el proceso de alfabetización inicial: un estudio con niños de tercero de preescolar
}

\author{
The influence of a computing resource on the initial literacy process: \\ A study with third grade preschool children \\ Gabriela Calderón Guerrero \\ Elizabeth Pohls Díaz
}

\begin{abstract}
RESUMEN
Se parte de una visión constructivista sobre la adquisición de la escritura. Diseño de la investigación: cuasi-experimental con alcance descriptivo y correlacional. Objetivo: determinar si el recurso informático propuesto (software educativo) favoreció significativamente la alfabetización inicial. Se trabajó con veinte niños de tercero de preescolar de una escuela pública mexicana. Se formó un grupo experimental y uno control, cuidando que fueran similares en diversos aspectos, incluyendo el nivel de conocimiento sobre el sistema de escritura. Ambos grupos fueron evaluados en torno a sus niveles de escritura al inicio (para formar los grupos experimental y control), a la mitad y al final de la investigación. El grupo experimental, durante un lapso de cuatro meses, dos veces por semana (20 minutos por sesión), trabajó con el software, mientras el grupo control no. Resultados: antes de utilizar el software ambos grupos mostraban los mismos niveles de escritura; a partir de la segunda y de la tercera evaluación, cuando el grupo experimental empezó a emplear el software, se observó una diferencia significativa a su favor. Los resultados sugieren que el uso de las nuevas tecnologías, con planteamientos didácticos precisos, favoreció el proceso de alfabetización inicial en la población estudiada.

Palabras clave: constructivismo, desarrollo, enseñanza, programa informático didáctico, sistema de escritura.
\end{abstract}

\begin{abstract}
The frame of reference that supports this research is the constructivist one. Research design: quasi-experimental with descriptive and correlational scope. Aim: to determine if the proposed computing resource (educational software) significantly encouraged the initial literacy. 20 third-year preschool children from a Mexican public school were interviewed. Experimental and control groups were formed, taking care that they were similar in various aspects, including their knowledge about the writing system. Both groups were evaluated on their knowledge about the writing system at the beginning (to form the experimental and control groups), in the middle and at the end of the research. The experimental group, during a period of four months twice a week (20 minutes per session), worked with the software, while the control group did not. Results: before using the software, both groups showed the same writing levels; from the second and third evaluation, when the experimental group started using the software, a significant difference was observed between both groups, favoring the experimental group. The results suggest that the use of new technologies, with precise didactic approaches, favored the initial literacy process of the population studied.

Keywords: constructivism, development, teaching, didactic computing software, writing system.
\end{abstract}




\section{INTRODUCCIÓN}

Uno de los temas objeto de álgidas discusiones y acalorados debates es el relativo a qué sistema de enseñanza se debe adoptar para lograr que los niños del siglo XXI adquieran de forma eficaz los contenidos de los planes de estudio, particularmente los relacionados con la lengua escrita y las matemáticas. Adicionalmente la contingencia sanitaria por SARS-CoV-2 acentúo la importancia tanto del uso como de la investigación en torno a cómo materiales multimedia, recursos informáticos (software educativo) y tecnologías digitales están presentes en la vida cotidiana en general y en los sistemas educativos en particular.

Por otra parte, resulta de especial interés el análisis e implementación de propuestas didácticas exitosas para el nivel de preescolar ya que hay suficiente consenso en que la calidad en este nivel educativo guarda una estrecha relación con el éxito académico y socio-afectivo de las personas en etapas posteriores (Bownan, Donovan y Burns, 2004; Dickinson, 2006; Guevara, López, García, Delgado y Hermosillo, 2008; NICHD, 2005; Rolla y Rivadeneira, 2006). Se sabe que los niños que en el preescolar han interactuado de forma sistemática con una amplia variedad de tipos de textos, así como prácticas sociales de lectura y escritura en entornos significativos, estarán en mejores condiciones para abordar y disfrutar de la escritura y la lectura de forma sistemática en los años venideros (Melhuish, Sylva, Sammons, Siraj-Blatchford, Taggart y Phan, 2008; Miranda, Ruíz, Vega y Miranda, 2017; Orellana y Melo, 2014; Snow, Burns y Griffin, 1998).

Las evaluaciones nacionales en materia de Lenguaje y comunicación realizadas en preescolar en un lapso de diez años (2007-2017), que se presentan en la tabla 1, indican un incremento de 17 puntos porcentuales en el nivel "debajo de básico" y un decremento en casi 13 puntos porcentuales en el nivel "avanzado" del año 2017 respecto al 2011; estos datos señalan fluctuación y poca consistencia de los resultados, por lo que urge desarrollar estrategias didácticas y de enseñanza más contundentes. Solo de esta forma se podrá cumplir con uno de los propósitos centrales planteados

Gabriela Calderón Guerrero. Profesora-investigadora de la Universidad Autónoma de Querétaro, México. Es licenciada en Psicología Educativa, especialista en Psicología de la Infancia y Aprendizajes Escolares; maestra en Psicología Educativa y doctora en Lingüística. Cuenta con diversas investigaciones y publicaciones nacionales e internacionales sobre temas como la construcción del conocimiento en la infancia y adolescencia, la adquisición de la lengua escrita y procesos cognoscitivos y lingüísticos. Así mismo colabora con la Biblioteca Infantil Universitaria de la UAQ. Cuenta con el perfil ProdeP y es miembro del Sistema Nacional de Investigadores, Nivel 1. Correo electrónico: gcalderonguerrero@gmail.com. ID: https://orcid.org/0000-0002-3725-6101.

Elizabeth Pohls Díaz. Universidad Autónoma de Querétaro, México. Es licenciada en Educación Preescolar, especialista en Enseñanza y Aprendizajes Escolares; maestra en Educación y actualmente estudiante del doctorado en Innovación Tecnológica Educativa por la UAQ. Cuenta con el diplomado en "Competencia lectora, un enfoque para la vida y el aula" por el ITESM. Actualmente es supervisora de educación preescolar de la zona 34 del estado de Querétaro y catedrática de la licenciatura en Pedagogía. Correo electrónico: elipohls@hotmail.com. ID: https://orcid.org/0000-0002-3107-3363. 
para preescolar por la Secretaría de Educación Pública en el documento Aprendizajes clave para la educación integral (SEP, 2017): "Desarrollar interés y gusto por la lectura, usar diversos tipos de texto e identificar para qué sirven; iniciarse en la práctica de la escritura y reconocer algunas propiedades del sistema de escritura” (p. 164).

Tabla 1. Porcentajes de logro alcanzado por alumnos del nivel preescolar en las pruebas Excale y Planea 2007-2017 en el área Lenguaje y Comunicación.

\begin{tabular}{cccccc}
\multirow{2}{*}{$\begin{array}{c}\text { Prueba/año } \\
\text { de aplicación }\end{array}$} & $\begin{array}{c}\text { Grado escolar } \\
\text { evaluado }\end{array}$ & Por debajo de básico & Básico & Medio & Avanzado \\
\hline Excale 2007 & Preescolar & $9 \%$ & $45 \%$ & $31 \%$ & $15 \%$ \\
\hline Excale 2011 & Preescolar & $6 \%$ & $42 \%$ & $34 \%$ & $17 \%$ \\
\hline PLANEA 2017 & Preescolar & $23 \%$ & $25,9 \%$ & $47,2 \%$ & $3,9 \%$ \\
\hline
\end{tabular}

Fuente: Elaboración propia con base en INEE (2011) e INEE (2018).

\section{Alfabetización INICIAL Y PSICOGÉNESIS DE LA LENGUA ESCRITA}

Desde una perspectiva constructivista de tipo psicogenético, la alfabetización inicial es la apropiación personal de un sistema de escritura que se expresa en la reconstrucción y reconocimiento de elementos, relaciones, convenciones, propiedades y características de dicho sistema (Ferreiro, 2013; Kaufman y Lerner, 2015; Molinari y Corral, 2008).

En ese sentido, las investigaciones psicogenéticas sobre el proceso de adquisición del sistema de escritura en la edad infantil (Calderón, 2005; Carrasco, Macías y López, 2017; Dueñas, 2017; Ferreiro, 2009, 2013; Ferreiro y Zamudio, 2008; Ferreiro y Teberosky, 2005; Grunfeld y Molinari, 2017; Grunfeld y Siro, 1997; Kaufman y Lerner, 2015; Molinari y Ferreiro, 2007; Quinteros, 1997; Teruggi y Molinari, 2015; Vernon, 1991, 1997 y 2004) han puesto énfasis en la importancia que tienen las actividades a las que el niño tiene acceso, las cuales deben cumplir con la característica de constituirse en retos cognitivos con el fin de permitir la estructuración y reestructuración de los conocimientos.

Los niveles de conceptualización de la lengua escrita, según la perspectiva psicogenética, se presentan siempre en el mismo orden de evolución, aunque las edades pueden variar, y son los siguientes:

1) Escrituras presilábicas. Se caracterizan por representaciones que no tienen por objetivo la búsqueda de la correspondencia entre los elementos que componen la pauta sonora y los elementos que constituyen la escritura. Las escrituras muestran aquellas características del sistema que los niños han hecho conscientes: diferencia entre dibujo y escritura, direccionalidad, arbitrariedad y uso de símbolos, pseudoletras o letras para representar algo por escrito.

2) Escrituras silábicas. El todo (oral y escrito) empieza a ser analizado, fragmentado, iniciándose así la búsqueda de correspondencias entre la pauta sonora 
(recorte silábico) y la escritura (una letra o pseudoletra): cada sílaba de lo oral es representada por una grafía o pseudografía escrita.

En este nivel empiezan a integrarse los valores sonoros convencionales. Estos se refieren a la consideración de que cierta letra representa cierto sonido y dicha representación empieza a coincidir con la convencional.

3) Escrituras silábico-alfabéticas. El niño descubre que la sílaba puede ser analizada, logrando en algunas ocasiones recortes fonémicos o bien recortes silábicos (en el plano oral); ambos recortes serán representados por una grafía o pseudografía.

4) Escrituras alfabéticas. Cuando los niños logran hacer de forma sistemática los recortes intrasilábicos fonémicos (en el plano oral), empiezan a representar, también sistemáticamente, ese fonema a través de una grafía (en el plano escrito) (Ferreiro y Teberosky, 2005), es decir, han conquistado el principio alfabético (una grafía representando de forma sistemática un fonema).

\section{RECURSOS INFORMÁTICOS: DESAFÍOS Y POSIBILIDADES}

Diversos estudios han mostrado la importancia y apuntalamiento de las tecnologías de la información y la comunicación (TIC) y de las tecnologías multimedia para el aprendizaje de distintos contenidos (Chiappe y González, 2014; Costa, Domenicantonio y Vacchino, 2010; Elizondo, Paredes y Prieto, 2006; Falcó, 2014; Falcón, 2012; González, Lozano y Ramírez, 2011; Hernández y Gutiérrez, 2016; Leal y Arias, 2009; León y Gómez, 2007; Pruzzo, 2005; Vidales, 2007), entre otras razones porque pueden favorecer la motivación e interés de los sujetos, además de potenciar el aprendizaje activo y el trabajo colaborativo a través de representaciones altamente significativas (Spriro, Feltovich, Jacobson y Coulson, 1991) que pueden ser procesadas por distintos mecanismos de memoria simultáneamente -teoría dual del almacenamiento cognitivo- (Latapie, 2007).

En relación específicamente al software educativo, como recurso informático, Guzmán, Ghitis y Ruiz (2009) señalan en su estudio que cuando se emplea con fines pedagógicos favorece la motivación de los sujetos, lo que potencialmente coadyuva al proceso de alfabetización inicial incluso con poblaciones que presentan déficit cognoscitivo leve, como es el caso de la población con la que trabajaron. Otros trabajos didácticos en el campo de la enseñanza matemática (Guedez, 2005; Sarmiento y Luna, 2017; Williner, 2011) coinciden con esta visión.

A nivel internacional diversos organismos han emitido sugerencias para la introducción de la tecnología al aula, incluso antes de la contingencia sanitaria por SARS-CoV-2. Según Hernández y Gutiérrez (2016), entre dichos organismos destaca la Organización para la Educación, la Ciencia y la Cultura de las Naciones Unidas (UNESCO). En el caso de México, el Programa Sectorial para la Educación 2020- 
2024 (SEP, 2020) establece como parte de sus objetivos y estrategias prioritarias la necesidad de brindar formación de calidad a los estudiantes apoyándose en las TIC.

El verdadero reto no consiste únicamente en equipar a las escuelas de diferentes dispositivos tecnológicos, sino especialmente en trasformar las prácticas didácticas de los docentes, así como las concepciones que se tienen sobre el sujeto que aprende y sobre el objeto de conocimiento, y que se encuentran en la base de todas las decisiones didácticas, incluidas las que son mediadas por las TIC. Al respecto, Lerner (2012) plantea lo siguiente: "La inclusión de esta herramienta (software) no genera automáticamente beneficios para el aprendizaje. Es imprescindible crear ciertas condiciones didácticas" (p. 55).

Un recurso informático que verdaderamente aspire a constituirse en aliado en el proceso de alfabetización inicial tendrá que proponer actividades que permitan a los niños reflexionar, aprender, equivocarse y volver a plantear hipótesis y preguntas sobre la escritura (Kriscautzky, 2012).

La presente investigación se centra en indagar si el uso de un recurso informático tipo software educativo, como recurso didáctico, puede apoyar el proceso de alfabetización inicial en las aulas. En ese sentido, el objetivo de la investigación fue constatar la influencia o no del recurso informático propuesto en el proceso de alfabetización inicial (logro del principio alfabético) de niños de tercero de preescolar.

\section{Diseño DE LA INVESTIGACIÓN}

La investigación tiene un diseño de tipo cuasi-experimental con un alcance descriptivo y correlacional en el que los constructos o variables de intervención fueron: uso (o no) del recurso informático educativo y el nivel de escritura alcanzado por los niños a lo largo de los tres momentos en que fueron evaluados. Los indicadores fueron: hipótesis presilábica, silábica, silábica-alfabética y alfabética (véase "Alfabetización inicial y psicogénesis de la lengua escrita”).

\section{Participantes}

Se seleccionaron 20 estudiantes (10 para formar el grupo experimental y 10 para el grupo control) de un jardín de niños público de la ciudad de Querétaro, Qro., México, cuyas edades oscilaban entre los 5 y los 6 años y que se encontraban cursando el tercer grado de educación preescolar en el mismo salón, es decir, los 20 niños tenían a la misma profesora que les impartía clases. El grupo experimental se constituyó de la mitad niños y la mitad niñas, el grupo se conformó por seis niños y cuatro niñas.

Se aplicó una primera evaluación de escritura (en adelante entrevista clínica piagetiana) para asegurar que los niños seleccionados contaran con un nivel de escritura similar entre ellos al iniciar la investigación y así configurar la distribución del grupo experimental con respecto del grupo control. 
Se eligió tercero de preescolar con el propósito de que los participantes contaran con acercamiento escolar previo a la cultura escrita; si bien no se esperaba que leyeran y escribieran de forma convencional y autónoma, sí se necesitaba que contaran con experiencias sistemáticas previas relativas a la exploración, producción e interpretación de diversos tipos de textos (aprendizaje esperado al egresar de preescolar) (SEP, 2017), situación que se constató en entrevista con la profesora del grupo.

Los criterios de inclusión de los participantes fueron:

a) Todos los participantes debían ser monolingües.

b) Que su rendimiento escolar fuera considerado, por la institución educativa, como promedio.

c) Que las condiciones socio-económicas y de escolaridad de las familias de los niños también fueran semejantes, según el reporte que la misma institución educativa y que la maestra de grupo proporcionó.

d) Que firmaran los padres o tutores el consentimiento informado en el que se notificaba que no se revelarían los nombres completos de los alumnos, ni la institución en la que estudiaban, entre otros datos sensibles y personales (como nombre completo con apellidos); únicamente se podrían usar los nombres de pila en los reportes de investigación, puesto que es información necesaria para la consecución de la misma.

El preescolar es una escuela de organización completa, es decir, con directora, docentes y apoyo administrativo. Fue elegida por ser una institución pública y urbana, así como por las facilidades y apertura que ofrecía para llevar a cabo el trabajo. Sin embargo, como institución no se caracterizaba por un trabajo sistemático y funcional con la lengua escrita, salvo la profesora del grupo escolar elegido, quien, cabe señalar, contaba con una formación de tipo constructivista en didáctica de la lengua escrita y las matemáticas.

\section{Procedimiento}

\section{Instrumento de recolección de datos}

Se aplicó la entrevista clínica piagetiana para conocer el nivel de escritura de cada participante a lo largo de la investigación. El instrumento empleado como evaluación se basa en el método clínico-crítico de la psicología genética y tiene el propósito de recuperar los datos necesarios para establecer la psicogénesis de las ideas infantiles a través de una interacción permanente entre las preguntas del investigador y las respuestas de los niños (García y Castorina, 2014).

La entrevista clínica piagetiana, en sus tres momentos de desarrollo, se llevó a cabo de forma individual y su aplicación tomó alrededor de 20 minutos (véase "Organización y encuadre de la investigación"). Se dictaba una serie de sustantivos (de cuatro, tres, dos y una sílaba) y se pedía al participante que fuera escribiendo lo que 
se le indicaba. En cuanto escribía cada palabra, se solicitaba la lectura (interpretación) de su escritura y se sostenía un diálogo para determinar la solidez de los esquemas de conocimiento. Antes de iniciar con el dictado de los sustantivos comunes, se pedía al participante que escribiera su nombre propio, ya que podía constituir referente para las escrituras que a continuación se le solicitarían. Una vez escrito el nombre propio, se solicitaba la escritura de siete sustantivos y una oración: foca, gato, gusano, perico, mariposa, sol, aro, el gato bebe leche.

Los sustantivos empleados poseen estructuras silábicas distintas. Se tienen dos palabras bisílabas CV-CV (consonante-vocal) y dos trisílabas CV-CV-CV. Esto responde al hecho de que en español la frecuencia más alta de palabras de contenido responde a estas dos estructuras silábicas. También se incluyó un tetrasílabo $\mathrm{CV}-\mathrm{CV}-\mathrm{CV}-\mathrm{CV}$, un monosílabo CVC, un bisílabo V-CV, todas palabras de contenido pleno, así como una oración.

Las producciones escritas de los niños fueron analizadas por el equipo de investigación para evaluar el nivel de escritura que presentaban. Los indicadores considerados fueron: nivel presilábico, silábico, silábico-alfabético y alfabético.

\section{Instrumento de intervención: el recurso informático (software educativo)}

El recurso informático con el que se trabajó fue diseñado por una de las integrantes del equipo de investigación que es docente de preescolar y cuenta con sólido conocimiento en didáctica constructivista y didácticas específicas.

Cabe señalar que el software empleado constituye el primer prototipo. Fue desarrollado, en los aspectos técnicos, por un ingeniero en sistemas, con apoyo de una diseñadora gráfica quien se encargó de las imágenes. El diseño del mismo giró en torno al contenido propuesto por las investigadoras, por lo que, teniendo al centro al niño y al objeto de conocimiento, se optó por el uso de tecnologías que permitieran la adaptabilidad a las necesidades que presentaran los alumnos, siempre considerando las características de la escritura como sistema alfabético y sistema de comunicación social. En términos técnicos, si bien el software no cuenta con herramientas de última generación, permitió evaluar el impacto de las actividades didácticas que constituyen su columna vertebral; se planea desarrollar en un corto plazo un software que funcione como una aplicación web cliente-servidor donde se diseñen las actividades con mayor interactividad, lo que a su vez genere una experiencia más atractiva para los usuarios, siempre teniendo como elementos fundamentales al sujeto cognoscente y al objeto de conocimiento.

La propuesta del software, en términos teórico-metodológico-didácticos, se basa en el trabajo de Vernon y Alvarado (2001); su diseño implicó, como ya se indicó, generar actividades didácticas sobre la lengua escrita en un ambiente digital y su mayor reto fue proponer en ese formato situaciones cognitivamente demandantes, lúdicas y susceptibles de apoyar a niños en distintos niveles de escritura. 
El software cuenta con siete tipos de actividades, todas ellas tendientes a favorecer el conocimiento del sistema de escritura y que se relacionan con los aprendizajes esperados para tercer grado de preescolar (que se comparten con primero y segundo de preescolar) (SEP, 2017) en el área de Lenguaje y comunicación, aprendizajes esperados que se desglosan y ejemplifican en la tabla 2.

Tabla 2. Aprendizajes esperados para tercero de preescolar y su correspondencia con las actividades del software educativo para alfabetización inicial.

\begin{tabular}{|c|c|c|c|}
\hline Organizador curricular 1 & Organizador curricular 2 & Aprendizajes esperados & Actividades del software \\
\hline Participación social & $\begin{array}{l}\text { Uso de documentos que } \\
\text { regulan la convivencia }\end{array}$ & $\begin{array}{l}\text { - Escribe su nombre con diversos propósitos } \\
\text { e identifica el de algunos compañeros } \\
\text { - Identifica su nombre y otros datos personales } \\
\text { en diversos documentos }\end{array}$ & $\begin{array}{l}\text { - Nombre propio } \\
\text { - Letras justas } \\
\text { - Unir palabras } \\
\text { - Completar palabras } \\
\text { - Memorama }\end{array}$ \\
\hline Participación social & $\begin{array}{l}\text { Producción e } \\
\text { interpretación de una } \\
\text { diversidad de textos } \\
\text { cotidianos }\end{array}$ & $\begin{array}{l}\text { - Interpreta instructivos, cartas, recados y } \\
\text { señalamientos } \\
\text { - Escribe instructivos, cartas, recados y } \\
\text { señalamientos utilizando recursos propios } \\
\text { - Produce textos para informar algo de interés a } \\
\text { la comunidad escolar o a los padres de familia }\end{array}$ & $\begin{array}{l}\text { - Colorear leyendo } \\
\text { - Completar palabras } \\
\text { - Niños alfabetizados: } \\
\text { a) inserta espacios } \\
\text { b) dónde dice } \\
\text { c) cosas para... }\end{array}$ \\
\hline
\end{tabular}

Fuente: Elaboración propia con base SEP, 2017, p. 198.

El recurso informático fue instalado en computadoras portátiles para que los niños trabajaran. La mitad de las computadoras fue provista por el equipo de investigadores, la otra mitad pertenecía al aula de medios de la escuela.

A continuación se describe un par de ejemplos de los tipos de actividades que presenta el recurso informático o software educativo:

- "Nombre propio". El nombre les da información a los niños en torno a que la escritura representa significados a través de ciertas formas escritas (palabras). Poco a poco los niños logran aprender que su nombre se representa de cierta manera. Posteriormente son capaces de comparar la escritura de su nombre con las de otros niños, lo cual les permite ir ampliando su repertorio gráfico y ampliar también las reflexiones en torno a cómo funciona el sistema de escritura.

En la actividad del software los niños tienen la oportunidad de escribir su nombre o el de algún compañero en un primer momento con referente y después sin este; un ejemplo de la actividad en el ambiente del software se muestra en la figura 1. 


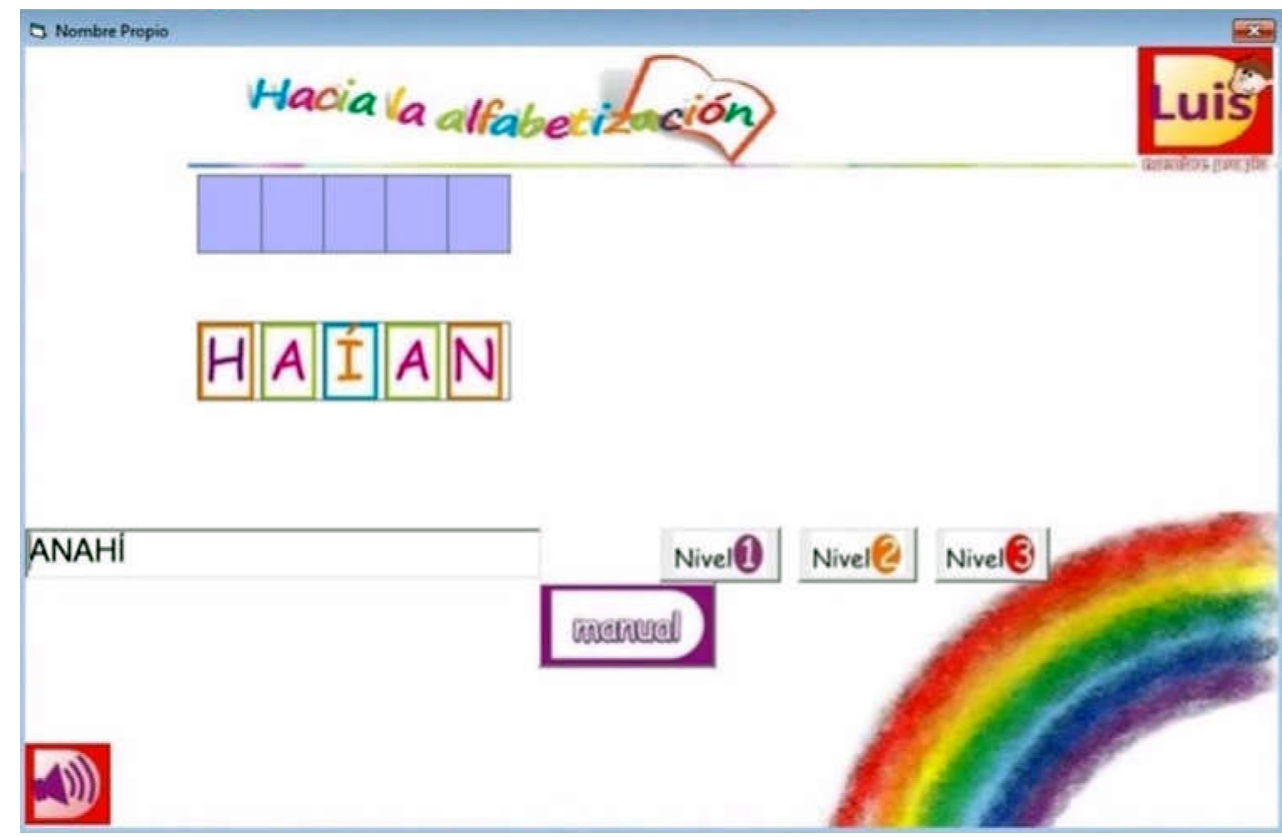

Figura 1. Actividad "Nombre propio" (ejemplo).

Fuente: Elaboración propia.

- “Letras justas". El propósito consiste en que los niños identifiquen, anticipen e interpreten qué palabra podrá formarse con las letras iniciales, finales e intermedias. Al trabajar con letras intermedias los niños reflexionan en torno a que la mayoría de las palabras están constituidas por más de una sílaba.

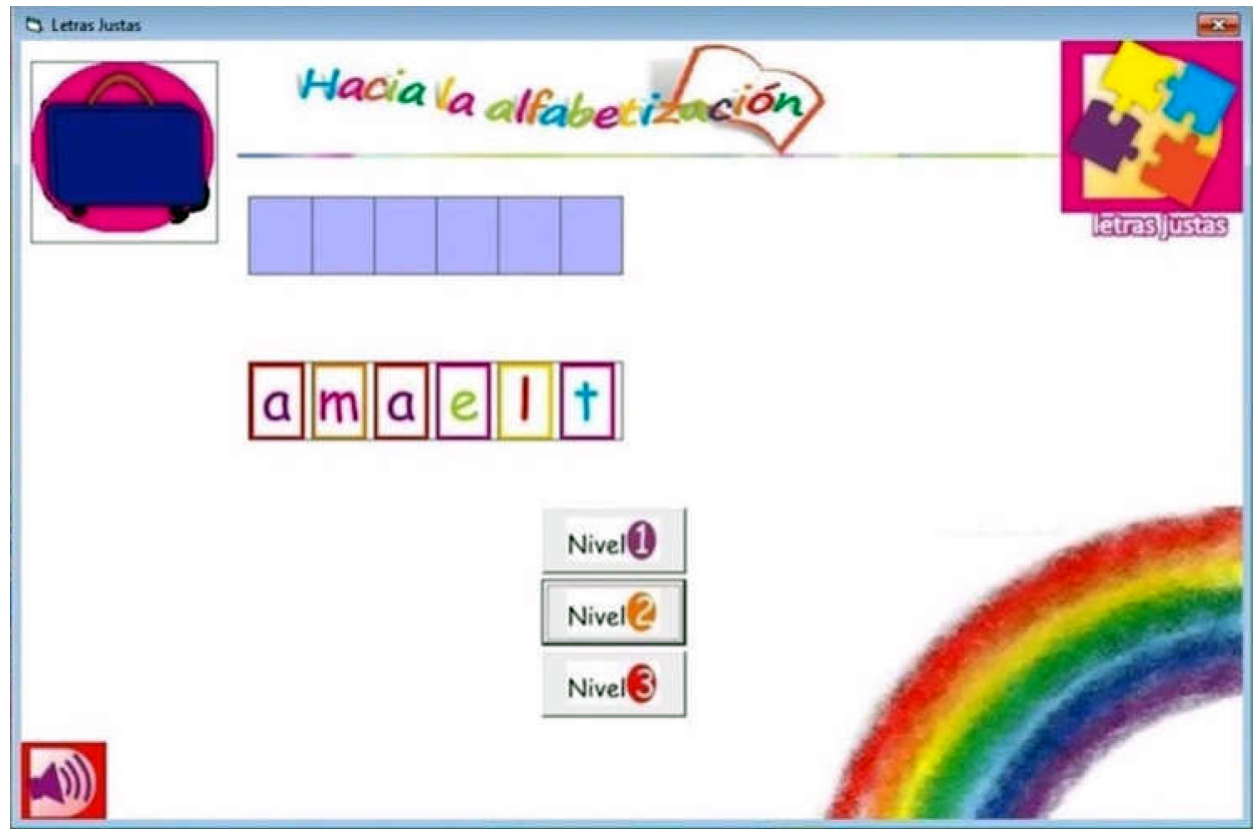

Figura 2. Actividad "Letras justas" (ejemplo).

Fuente: Elaboración propia. 
En la actividad de "Letras justas" (figura 2) se le proporcionan al niño las letras exactas, en desorden, para formar una palabra, la cual tendrá que ir acomodando. La complejidad de la palabra varía según el nivel de conocimiento de los niños sobre el sistema.

Todas las actividades, a excepción de "Niños alfabetizados", tienen tres niveles de complejidad los cuales se jugarán en función del avance de los alumnos; las consignas cuentan con audio para que el niño en momentos muy iniciales pueda saber qué debe hacer. Las consignas son cortas y claras.

\section{ORgANIZACIÓN Y ENCUADRE DE LA INVESTIGACIÓN}

La organización de los momentos de aplicación de las entrevistas y de la intervención se presenta en la tabla 3 .

Tabla 3. Organización y encuadre de las actividades llevadas a cabo en la investigación.

\begin{tabular}{|c|c|c|c|c|}
\hline $\begin{array}{c}\text { Primera entrevista } \\
\text { clínica } \\
\text { (evaluación) }\end{array}$ & $\begin{array}{l}\text { Primer periodo de } \\
\text { intervención con el } \\
\text { recurso informático }\end{array}$ & $\begin{array}{c}\text { Segunda entrevista } \\
\text { clínica } \\
\text { (evaluación) }\end{array}$ & $\begin{array}{l}\text { Segundo periodo de } \\
\text { intervención con el } \\
\text { recurso informático }\end{array}$ & $\begin{array}{c}\text { Tercera entrevista } \\
\text { clínica } \\
\text { (evaluación) }\end{array}$ \\
\hline \multicolumn{5}{|l|}{ Grupo experimental } \\
\hline $\begin{array}{l}1 \text { sesión individual de } \\
20 \text { minutos (principios } \\
\text { de septiembre) }\end{array}$ & $\begin{array}{c}16 \text { sesiones } \\
\text { (septiembre/octubre): } \\
20 \text { minutos } \\
\text { cada sesión }\end{array}$ & $\begin{array}{l}1 \text { sesión individual } \\
\text { de } 20 \text { minutos } \\
\text { (finales de octubre) }\end{array}$ & $\begin{array}{l}16 \text { sesiones (noviembre/ } \\
\text { diciembre/enero): } \\
20 \text { minutos cada sesión }\end{array}$ & $\begin{array}{l}1 \text { sesión individual } \\
\text { de } 20 \text { minutos (enero) }\end{array}$ \\
\hline \multicolumn{5}{|l|}{ Grupo control } \\
\hline $\begin{array}{c}1 \text { sesión individual de } 20 \\
\text { minutos (principios de } \\
\text { septiembre) }\end{array}$ & - & $\begin{array}{l}1 \text { sesión individual } \\
\text { de } 20 \text { minutos } \\
\text { (finales de octubre) }\end{array}$ & & $\begin{array}{l}1 \text { sesión individual } \\
\text { de } 20 \text { minutos (enero) }\end{array}$ \\
\hline
\end{tabular}

Fuente: Elaboración propia.

Se decidió iniciar la aplicación a inicios del ciclo escolar (septiembre) ya que se consideró que así se tendría tiempo suficiente para la intervención completa con el recurso informático. En total la intervención se llevó a cabo a lo largo de cuatro meses. Las sesiones de intervención con el recurso informático (grupo experimental) se llevaron a cabo dos veces a la semana en sesiones de 20 minutos.

La docente del grupo se encargó de apoyar en el uso del recurso informático a los niños en la primera sesión de intervención y posteriormente una asistente apoyó durante ocho sesiones a los niños, únicamente en el manejo técnico del recurso informático. Las sesiones fueron por las mañanas en los horarios en los que el grupo control tomaba clases relativas al área de Lenguaje y comunicación en un salón contiguo al del grupo control. 
Finalmente, se construyó una base de datos en la que se consignaron y procesaron, a través de pruebas de estadística no paramétrica, los resultados de ambos grupos en las tres evaluaciones que se aplicaron. ${ }^{1}$

\section{Resultados}

A continuación se exponen los resultados obtenidos de la comparación entre el grupo experimental versus el grupo control en cada una de las tres evaluaciones (aplicación de la entrevista clínica piagetiana) realizadas a lo largo de la investigación: al inicio, en la parte intermedia y al final del proyecto.

En la figura 3 se presentan los resultados obtenidos en la primera entrevista (evaluación).

La figura 3 indica que los niveles de escritura de ambos grupos eran muy similares al inicio de la investigación y, por lo tanto, los participantes partieron de condiciones prácticamente iguales respecto a su nivel de adquisición del sistema de escritura. Incluso en el grupo control se puede observar la presencia del nivel de escritura alfabético, que no se observa en el grupo experimental.

La mediana del grupo experimental y el control fue de 1,0 para ambos; los grupos no difieren significativamente entre sí (Mann-Whitney $U=35,5, n 1=n 2=20$, $P>0,05$ dos colas).

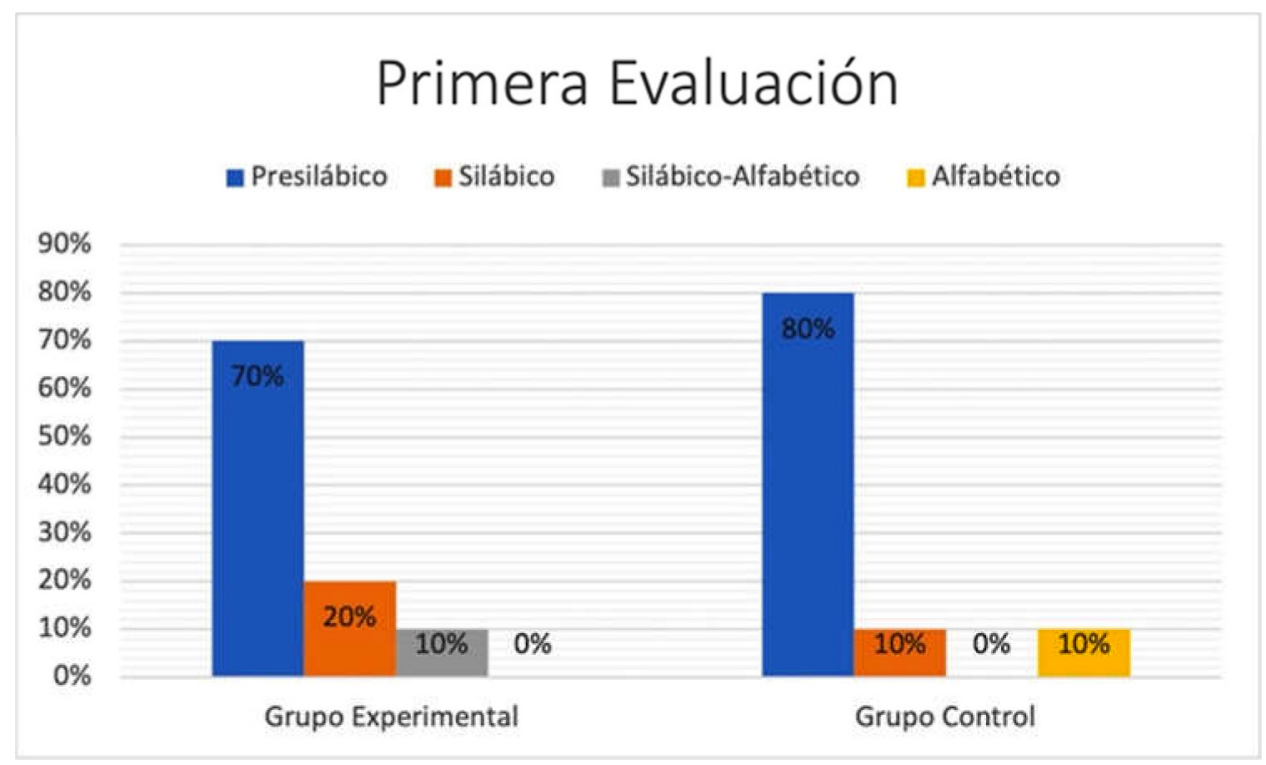

Figura 3. Porcentajes alcanzados por el grupo A y el grupo B en la primera prueba de escritura.

Fuente: Elaboración propia.

1 Se emplearon dos pruebas: primero la prueba Kolmogorov-Smirnov para determinar si la muestra presentaba o no una distribución normal; como no presentó una distribución normal, se optó por una prueba no paramétrica (U de Mann-Whitney), que se utiliza para calcular el valor de $Z$ en poblaciones pequeñas organizadas en dos grupos. 


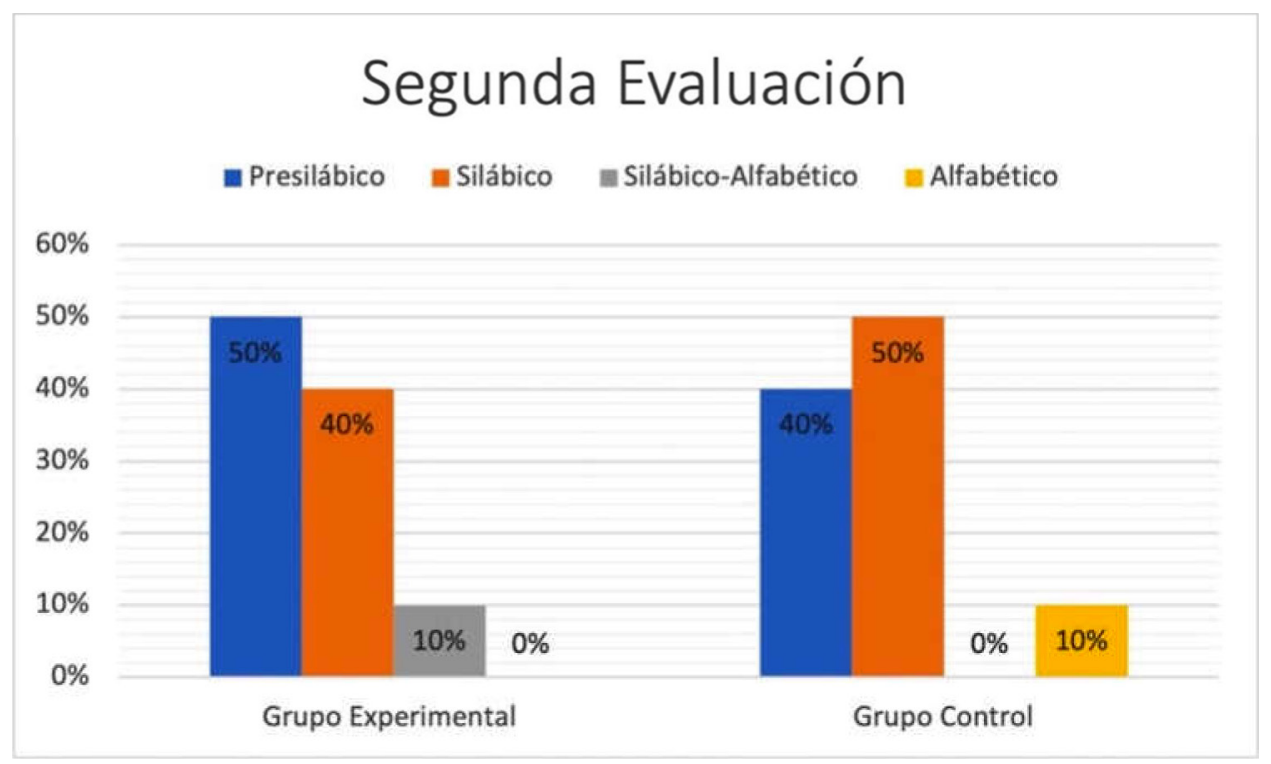

Figura 4. Porcentajes alcanzados por el grupo A y el grupo B en la segunda prueba de escritura.

Fuente: Elaboración propia.

Dos meses más tarde se aplicó la segunda prueba de escritura. En la figura 4 se muestran los porcentajes alcanzados por ambos grupos en ese momento.

Para ese momento, la mediana del grupo experimental y el grupo control fue de 3,0 y 2,0 respectivamente; los grupos difieren significativamente entre sí (MannWhitney $U=10,0, n 1=n 2=20, P<0,05$ dos colas).

Finalmente, en la última evaluación, los porcentajes alcanzados en los distintos niveles de conceptualización de la lengua escrita se señalan en la figura 5.

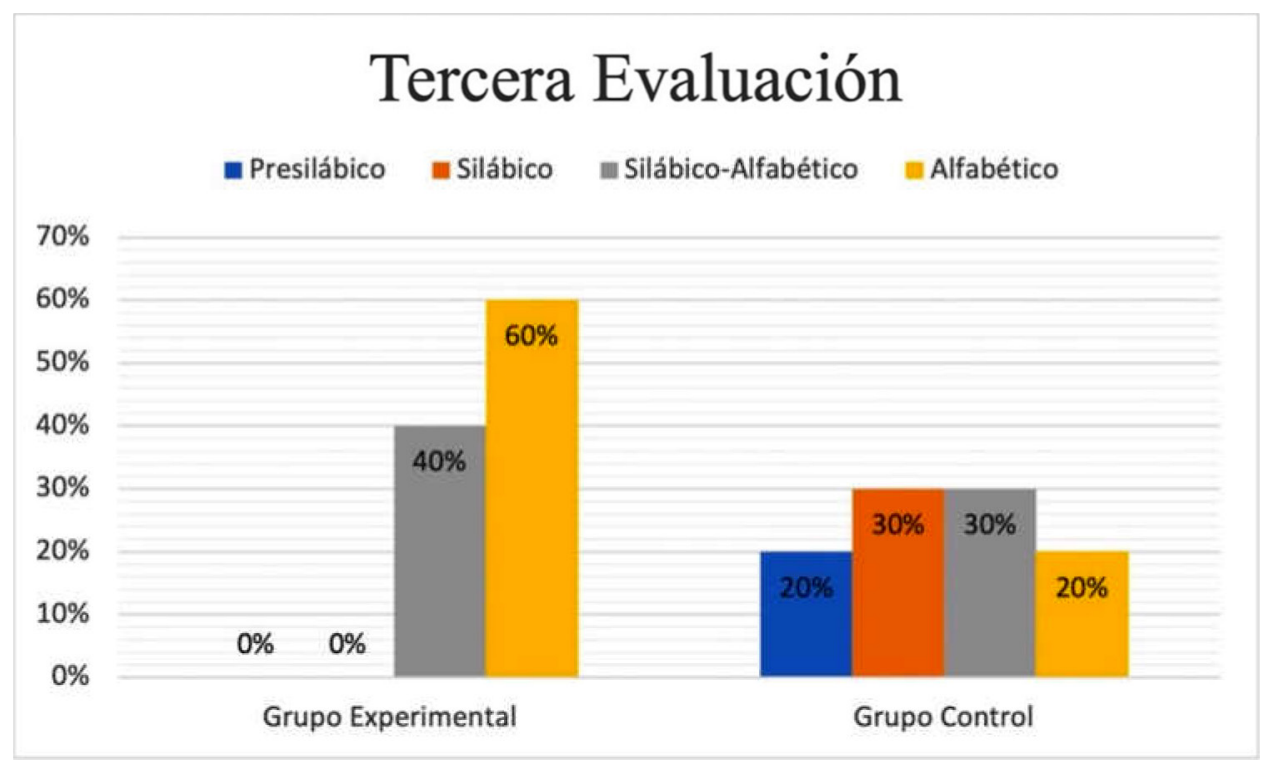

Figura 5. Porcentajes alcanzados por el grupo A y el grupo B en la tercera prueba de escritura. Fuente: Elaboración propia. 
La mediana alcanzada por el grupo experimental y el grupo control fue de 4,0 y 2,0 respectivamente; los grupos difieren significativamente entre ellos (Mann-Whitney $U=12,5, n 1=n 2=20, P<0,05$ dos colas).

A modo de ejemplo, se muestra la evolución de las producciones escritas de Diego a lo largo de la intervención. Este participante pertenecía al grupo experimental.

Cabe señalar que en cada una de las imágenes la escritura del niño es la que aparece al lado izquierdo de las hojas, seguida de la escritura normalizada de la entrevistadora para registrar lo que el niño había escrito, cuando esto fue necesario para comprender el texto del participante.

Como se aprecia en la figura 6, Diego inició mostrando una escritura presilábica: empleaba una cantidad más o menos fija de letras para representar cada una de las palabras que le fue dictada, entre cinco y siete grafías por palabra. También es posible observar un repertorio de letras variable, sin valor sonoro convencional, aunque empleando como base su nombre, cuyas letras usó como comodín, por ejemplo, en perico. Así mismo, la forma en que Diego leyó sus producciones en la primera evaluación fue con lectura global, es decir, sin ser capaz de segmentar ni el todo oral (la palabra oral en cuestión), ni el todo escrito (la palabra escrita).

Dos meses más tarde, Diego había avanzado, según la prueba de escritura, al nivel silábico-alfabético. Su escritura mostró ya un control de cantidad de letras en función de la segmentación del todo oral (a veces interpretado como sílaba, a veces como fonema). También se observa el uso del valor sonoro convencional de las letras para representar ciertos fonemas o sílabas. El monosílabo sol ya está representado con sus

a) Primera evaluación

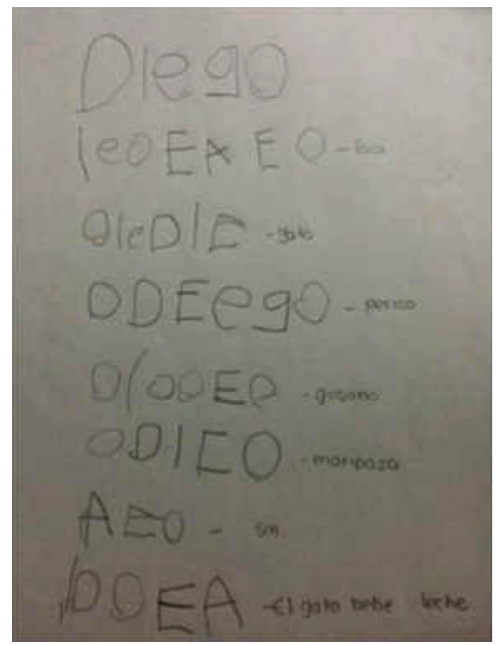

Hipótesis presilábica b) Segunda evaluación

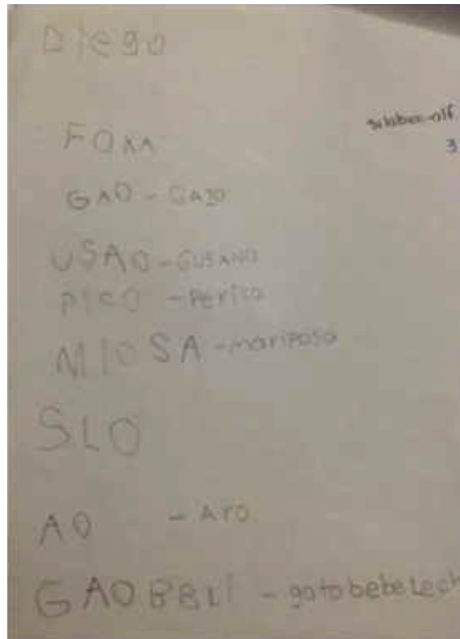

Hipótesis silábico-alfabética c) Tercera evaluación

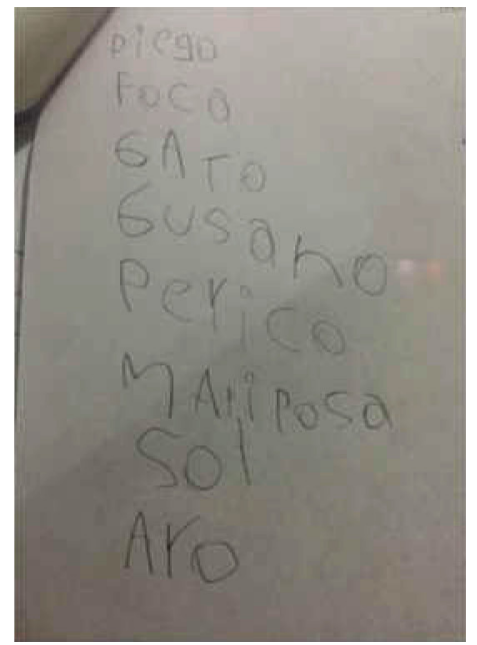

Hipótesis alfabética

Figura 6. Ejemplo de la evolución en torno a la conceptualización del sistema de escritura de un participante del grupo experimental.

Fuente: Elaboración propia. 
tres grafías, aunque aún no en el orden canónico. Aro, bisílabo de estructura vocalconsonante-vocal (V-CV) es una palabra difícil para los niños que están comprendiendo cómo opera el sistema de escritura ya que rompe la estructura clásica de las palabras de contenido del español que son bisílabos consonante-vocal-consonante-vocal (CV-CV); Diego la representó de la siguiente manera: A o y la leyó / A-RO/, haciendo corresponder la letra A con la A oralizada y la letra o con la sílaba oral Ro.

Para la última evaluación, Diego ya había avanzado a un nivel de escritura alfabético. Incluso ya no fue necesario que se escribiera lo que el participante quiso representar, dado que su escritura, al menos a nivel del principio alfabético, era totalmente convencional.

En la figura 7 se presentan las escrituras de Emma, participante del grupo control.

Emma, cuyas muestras de escritura y su evolución a lo largo de los tres momentos de la evaluación en la investigación se observan en la figura 7, inició con una escritura de tipo presilábica, con repertorio fijo (empleando básicamente las grafías de su nombre) y cantidad variable de letras que denota su imposibilidad de segmentar el todo oral (la palabra oral) del todo escrito (la palabra escrita) y de establecer una relación entre ambos (oral y escrito); esto se constató con el tipo de lectura de Emma hizo de sus producciones escritas: una interpretación global, señalando con su dedo la palabra escrita completa, y repitiendo completa también la palabra oral.

Dos meses más tarde, Emma había alcanzado un nivel de escritura silábico, con lo que ya era capaz de segmentar el todo oral (la palabra oral) en sílabas y representar cada sílaba de lo oral con una grafía de lo escrito. Se observa también la presencia

a) Primera evaluación

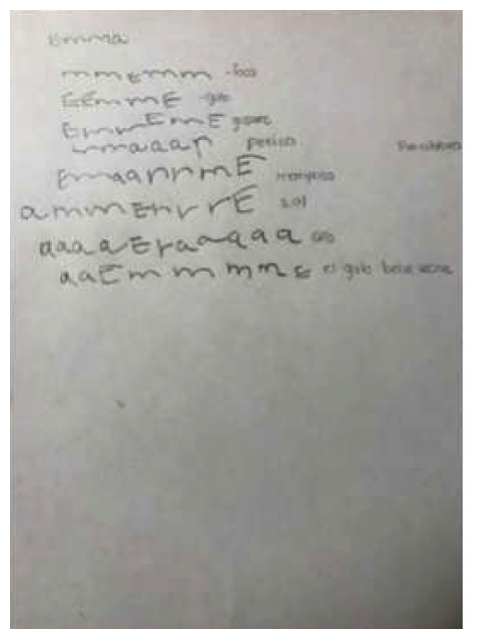

Hipótesis presilábica b) Segunda evaluación

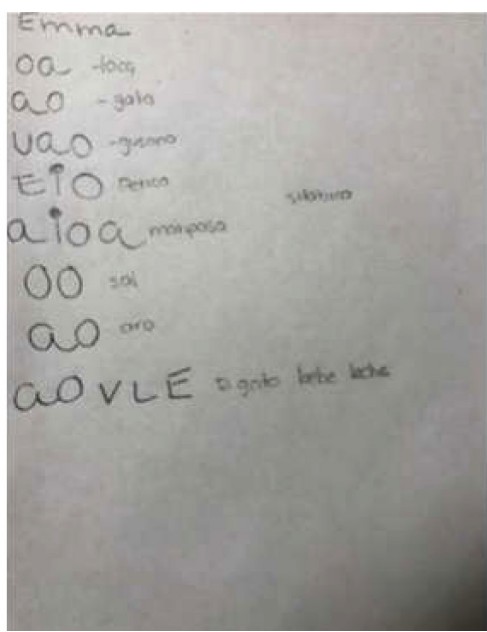

Hipótesis silábico-alfabética c) Tercera evaluación

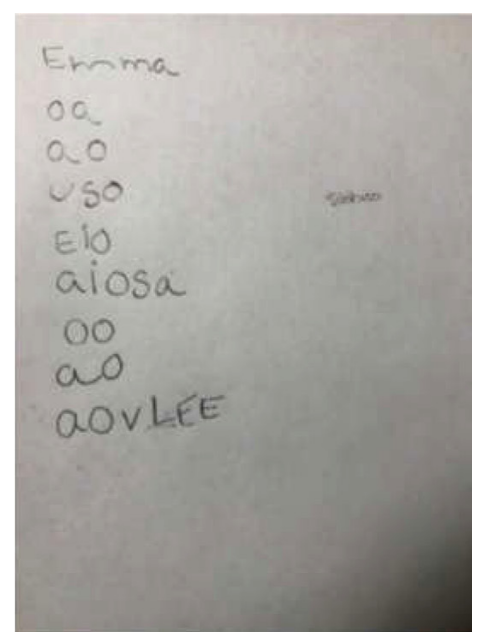

Hipótesis alfabética

Figura 7. Ejemplo de la evolución en torno a la conceptualización del sistema de escritura de un participante del grupo control.

Fuente: Elaboración propia. 
de valor sonoro convencional en la producción escrita de la participante en esta evaluación. En ese sentido, Emma logró un avance importante respecto a su primer tipo de escritura.

Para la tercera evaluación, Emma continuaba exhibiendo una hipótesis silábica estricta; es más, las escrituras de la segunda evaluación y las de la tercera son prácticamente idénticas, salvo por la palabra gusano que en la segunda evaluación fue escrita como U A O (leyendo en voz alta: GU-SA-NO y señalando cada una de las sílabas de lo oral por cada una de las grafías escritas) y en la tercera como u s o (leyendo en vOZ alta: GU-SA-NO y señalando cada una de las sílabas de lo oral por cada una de las grafías escritas).

\section{Comentarios Finales}

En este trabajo se han expuesto los resultados alcanzados por un grupo de participantes de tercero de preescolar en relación a la alfabetización inicial, empleando un recurso informático educativo. Para analizar los datos obtenidos y poder valorar los hallazgos de la investigación fue necesario construir una base de datos en la que se contrastaron los resultados alcanzados por el grupo experimental y el grupo control en la entrevista inicial, en la entrevista intermedia y en la entrevista final respecto a la conceptualización del sistema de escritura (que se apreciaba a partir del nivel de escritura alcanzado por los niños).

Nuestros resultados permiten afirmar que el recurso informático empleado favoreció una intervención didáctica exitosa: las pruebas estadísticas confirmaron una diferencia significativa al final de la intervención didáctica con el recurso informático, a favor de los niños del grupo experimental por sobre los del grupo control; si bien al inicio del trabajo ambos grupos mostraban conocimientos muy similares respecto a la escritura. Esto permite afirmar que el recurso informático tuvo un impacto positivo en la evolución de las concepciones en torno a la escritura de los niños del grupo experimental. Entre las razones por las cuales el recurso informático pudo ser tan favorecedor se encuentra su diseño, un diseño que parte de una posición teórico/ metodológica de tipo constructivista.

En el enfoque constructivista se propone al niño, a través del diseño de las actividades mismas, que la escritura es, antes que nada, un objeto de naturaleza social y por ende sirve para comunicar y comunicarse, para dejar registro de un pensamiento o una idea en un momento dado, para resolver problemas. Desde esta perspectiva, el niño avanza en su conocimiento de la escritura haciéndose preguntas y generando hipótesis; el software o recurso informático literalmente abre una serie de posibilidades para explorar, avanzar, retroceder, acertar, equivocarse, en un ambiente multimedia, atractivo y lúdico y en el que las consecuencias de sus decisiones lo retroalimentan. De esta manera, favorecer la alfabetización inicial a través de propuestas didácticas, 
bajo la perspectiva de la psicogénesis de la lengua escrita, puede tener en el uso de las nuevas tecnologías un gran aliado; sin embargo, para que esto sea posible es necesario proponer un recurso informático que, retomando las mejores propuestas constructivistas para la alfabetización, pueda llevarlas a un ambiente digital sin desvirtuarlas; consideramos que esta es la mayor aportación del prototipo de software cuyos resultados, en su aplicación, aquí hemos descrito. En ese sentido, los resultados de la investigación de Guzmán, Ghitis y Ruiz (2009), investigadoras que trabajaron también sobre la alfabetización empleando e-books, apuntalan los hallazgos del presente trabajo.

Por otro lado, es altamente probable que el software, al hacer uso de recursos multimedia, haya resultado divertido, lúdico, y por lo tanto haya favorecido la motivación y autorregulación de los niños (Guzmán, Ghitis y Ruiz, 2009), así como apuntalado el procesamiento de información apelando a diversos tipos de memoria. Sin embargo, será necesario llevar a cabo mayor investigación al respecto (Latapie, 2007).

En la sección introductoria, retomando los aportes de Lerner (2012) y Kriscautzky (2012) se apuntaba la necesidad de integrar las nuevas tecnologías (como la computadora y los recursos informáticos) a la educación, pero sin dejar de considerar que estas por sí mismas no resolverán los problemas y retos educativos. Es verdad que la tecnología que se emplee puede favorecer procesos cognoscitivos inusitados, pero para que sea así debe inscribirse en prácticas sociales, reales y significativas. Es decir, no se trata de hacer traducciones multimedia de viejas propuestas alfabetizadoras, que centran su atención en la motricidad y en la idea de sistema de escritura como código de transcripción y del niño como ente únicamente receptivo; insistimos en ello ya que diversos trabajos y propuestas didácticas, desarrolladas con el fin de favorecer la alfabetización inicial a través del uso de recursos tecnológicos, específicamente software, parten de una falta de conocimiento tanto de la naturaleza de la lengua escrita, como objeto de conocimiento y objeto social e histórico, como de las necesidades y características del sujeto que aprende, lo que genera que dichas propuestas pongan el énfasis en aspectos figurales como el trazo de las letras, la fonética de las vocales y las consonantes, así como en una concepción empobrecida del sistema de escritura y del niño, y se olviden de los aspectos conceptuales que son los verdaderamente relevantes en la construcción de este conocimiento. De esta forma, propuestas como las que recién hemos descrito tienden a ser más un recurso de entretenimiento que verdaderos apoyos didácticos que favorezcan que el niño avance en su conocimiento sobre la escritura, favorecer que se pregunte qué es lo que representa la escritura y cómo lo representa (Ferreiro, 1999).

En ese sentido, es necesario cuestionar el verdadero sentido y valor de ciertas propuestas y materiales didácticos empleados como medios para favorecer la alfabetización. Este análisis resulta urgente ya que muchos de los materiales -incluidos los digitales-actualmente utilizados en las aulas poco tienen que ver con las aportaciones que han hecho las investigaciones en este campo (Calderón, 2005; Carrasco, Macías 
y López, 2017; Dueñas, 2017; Ferreiro, 2009; Ferreiro y Zamudio, 2008; Ferreiro y Teberosky, 2005; Grunfeld y Molinari, 2017; Grunfeld y Siro, 1997; Kaufman y Lerner, 2015; Molinari y Ferreiro, 2007; Quinteros, 1997; Teruggi y Molinari, 2015; Vernon, 1991, 1997 y 2004) y que fueron insumos esenciales para el desarrollo de la presente investigación. Por otra parte, nuestros resultados, en términos generales, coinciden con planteamiento del trabajo antecedente de Guzmán, Ghitis y Ruiz (2009), trabajo con el cual, lejos de sostener un debate, coincidimos respecto a la conceptualización en torno al sistema de escritura y al sujeto cognoscente, si bien las autoras desarrollaron su investigación con una población con déficit cognitivo leve y no describieron las actividades alfabetizadoras.

Por otro lado, resulta necesario enfatizar que el recurso informático no sustituye la acción docente. Los resultados muestran que los niños del grupo control, al contar con el trabajo de su maestra que tenía una formación en didáctica constructivista de la lengua escrita, también avanzaron. Sin embargo, que el docente pueda apoyarse en su labor diaria en una herramienta de este tipo podría potenciar no solo a los niños sino la acción docente misma, al permitirle al profesor tener tiempo para centrarse en niños que requieran, por sus características personales, mayor apoyo de su parte o bien fijarse en aspectos de la escritura, el lenguaje y la cultura escrita que sería interesante destacar, mientras descansa parte del trabajo sobre el sistema de escritura en el recurso informático educativo.

La propuesta del presente trabajo y con la que concluimos es la siguiente: lo más importante es partir de un modelo sólido en torno a la construcción del conocimiento, ya que, si bien los niños lograrán eventualmente alcanzar el principio alfabético, si continúan en la escuela, ¿cómo se pretende que lo alcancen?, ¿qué harán con ese conocimiento?, ¿se sentirán/formarán parte de la cultura escrita?, ¿habremos sido capaces de transmitir que la escritura es valiosa para plantearse y resolver problemas de diversa índole?, ¿es suficiente con que los niños y niñas decodifiquen las letras o una buena propuesta didáctica alfabetizadora tendría que favorecer la formación de ciudadanos intérpretes y productores de texto que puedan ser parte activa de forma consciente y decidida de la toma de decisiones en su entorno social? Son sin duda muchas las preguntas y los retos. Sin embargo, este primer acercamiento con veinte niños permite pensar que quizá sea posible articular la dimensión lúdica con el favorecimiento de reflexiones precisas sobre el principio alfabético empleando la tecnología, con lo que se estará un paso más cerca de brindar a los niños y niñas, como sociedad y escuela, una educación más integral, significativa y ciudadana en torno a la escritura y la cultura escrita.

Finalmente, es absolutamente necesario trabajar de forma más comprometida y sistemática en una sólida formación de los docentes a propósito de didácticas específicas y apoyarlos con herramientas como lo son las TIC y recursos informáticos (software educativo) de cara a los retos del siglo XXI, retos tales como la contingencia 
sanitaria por SARS-CoV-2. Heriberto Yépez señalaba: "La computadora es un electrodoméstico. Sí, pero es el único electrodoméstico que ha cambiado el lenguaje" (citado en Raphael, 2011, p. 188). Hoy estas palabras resultan más ciertas y contundentes que nunca.

\section{REFERENCIAS}

Bowman, B., Donovan, S., y Burns, M. (2004). Eager to learn. Washington, DC, EE.UU.: National Academy Press.

Calderón, G. (2005). Análisis de lo oral y conocimiento del sistema de escritura en niños preescolares: un enfoque cualitativo [Tesis de Maestría]. Universidad Autónoma de Querétaro, Querétaro, México.

Carrasco, A., Macías, V., y López, G. (2017). Una experiencia de alfabetización inicial. Metodología, diseño didáctico y valoración docente. Ponencia presentada en el XIV Congreso de Investigación Educativa COMIE. San Luis Potosí, México. Recuperado de: http://www. comie.org.mx/congreso/memoriaelectronica/v14/ doc/2228.pdf.

Costa, A., Di Domenicantonio, M., y Vacchino, C. (2010). Material educativo digital como recurso didáctico para el aprendizaje del cálculo integral y vectorial. Revista Iberoamericana de Educación Matemática, (21), 173-185. Recuperado de: http://www.fisem.org/www/union/ revistas/2010/21/Union_021_018.pdf.

Chiappe, A., y Gonzalez, A.-R. (2014). Los procesadores de texto y los niños escritores: un estudio de caso. Revista Estudios Pedagógicos, (2), 101-115.

Dickinson, D. K. (2006). Cognitive and linguistic building blocks of early literacy. En D. K. Dickinson y S. B. Neuman (eds.), Handbook of early literacy research (vol. 2). Nueva York: Guilford Publications.

Dueñas, S. (2017). Descripción y análisis de actividades e intervenciones docentes para la alfabetización inicial bajo un enfoque constructivista [Tesis de Maestría]. Universidad Autónoma de Querétaro, Querétaro, México.

Elizondo, A., Paredes, F., y Prieto, A. (2006). Enciclomedia: un programa a debate. Revista Mexicana de Investigación Educativa, 11(28), 208-224. Recuperado de: http://www.redalyc.org/pdf/140/14002811.pdf.

Falcó, M. (2014). Un wiki-libro creado por los estudiantes para aprender matemáticas. Revista Científica de Opinión y Divulgación, 10(30), 1-13. Recuperado de: http:// dim. pangea.org/revistaDIM30/docs/AR30wikilibro.pdf.
Falcón, R. M. (2012). El ordenador portátil como herramienta de apoyo en el aprendizaje activo de matemática aplicada a la edificación. Revista de Medios y Educación, (40), 47-60. Recuperado de: http://www. redalyc.org/articulo.oa?id $=36823229004$.

Ferreiro, E. (1999). Alfabetización, teoría y práctica. México: Siglo XXI.

Ferreiro, E. (2009). Desestabilización de las escrituras silábicas: alternancia y desorden con pertinencia. Revista Lectura y Vida, 30(2), 6-13.

Ferreiro, E. (2013). El ingreso a la escritura y a las culturas de lo escrito. Textos de investigación. México: Siglo XXI.

Ferreiro, E., y Teberosky, A. (2005). Los sistemas de escritura en el desarrollo del niño (22a. ed.). México: Siglo XXI.

Ferreiro, E., y Zamudio, C. (2008). La escritura de sílabas CVC y CCV en los inicios de la alfabetización escolar. $¿$ Es la omisión de consonantes prueba de incapacidad para analizar la secuencia fónica? Rivista di Psicolinguistica Applicata, (8), 1-2. Recuperado de http://digital. casalini.it/10.1400/94557.

García, I., y Castorina, J. (2014). Método clínico-crítico y etnografía en investigaciones sobre conocimiento sociales. Cadernos de Pesquisa, 44(154), 1052-1068. Recuperado de http://dx.doi.org/10.1590/198053142949.

González, J., Lozano, F., y Ramírez, M. (2011). Uso de la tecnología en ambientes de aprendizaje de educación básica: ¿Por qué no basta con infraestructura tecnológica y capacitación? Memorias del XI Congreso Nacional de Investigación Educativa, México. Recuperado de: http://www. ruv.itesm.mx/convenio/catedra/recursos/material/ cn_22.pdf.

Grunfeld, D., y Molinari, M. (2017). La importancia de pensar sobre la escritura en el jardín de infantes. Magis, Revista Internacional de Investigación en Educación, 9(19), 31-48. Recuperado de https://doi.org/10.11144/ Javeriana.m9-19.ipej.

Grunfeld, D., y Siro, A. (1997). La palabra: complejas relaciones entre el todo y las partes. Programa de investigación: adquisición de la lengua escrita [colec. CEA-CBC]. Buenos 
Aires, Argentina: Universidad de Buenos Aires, Centro de Estudios Avanzados/Oficina de Publicaciones del Ciclo Básico Común.

Guedez, M. (2005). El aprendizaje de funciones reales con el uso de un software educativo: una experiencia didáctica con estudiantes de educación de la ULATáchira. Acción Pedagógica, (14), 38-49.

Guevara, Y., López, A., García, G., Delgado, U., y Hermosillo, A. (2008). Nivel de escritura en alumnos de primer grado, de estrato sociocultural bajo. Revista Perfiles Educativos, 30(121), 41-62.

Guzmán, J., Ghitis, T., y Ruiz, C. (2009). Uso de la tecnología en la alfabetización de niños con déficit cognitivo leve. Infancias Imágenes, 8(1), 25-31. Recuperado de https://doi.org/10.14483/16579089.4491.

Hernández, L., y Gutiérrez, D. (2016). Las tecnologías multimedia y su relación con el aprendizaje de la matemática en alumnos de sexto grado de educación primaria. Revista Educación y Ciencia, 5(45), 50-65.

INEE [Instituto Nacional para la Evaluación de la Educación] (2011). Exámenes de la calidad y el logro educativos. Tercero de preescolar, aplicación 2011. Bases de datos Lenguaje y comunicación. Recuperado de: https:// historico.mejoredu.gob.mx/evaluaciones/planea/ excale/tercero-preescolar-2010-2011/.

INEE (2018). Evaluaciones de logro referidas al sistema educativo nacional. 3er grado de preescolar, ciclo escolar 2017-2018. Bases de datos Lenguaje y comunicación. Recuperado de: https://www.inee.edu.mx/evaluaciones/planea/ base-de-datos-planea-aplicacion-a-tercer-grado-depreescolar-del-ciclo-escolar-2017-2018/.

Kaufman, A., y Lerner, D. (2015). Documento transversal No. 1. La alfabetización inicial. Buenos Aires: Ministerio de Educación de la Nación.

Kriscautzky, M. (2012). Prácticas de lectura y escritura en entornos digitales: la pertinencia de incluirlas desde la alfabetización inicial en las TIC en la escuela. En D. Goldin, M. Kriscautzky y F. Perelman (eds.), Las TIC en la escuela, nuevas herramientas para viejos y nuevos problemas (pp. 243-368). México: Océano Travesía.

Latapie, I. (2007). Acercamiento al aprendizaje multimedia. Revista Investigación Universitaria Multidisciplinaria, 6(6), 7-14. Recuperado de: https://www.google.com. $\mathrm{mx} / \mathrm{url}$ ? $\mathrm{sa}=\mathrm{t} \& \mathrm{rct}=\mathrm{j} \& \mathrm{q}=\& \mathrm{esrc}=\mathrm{s} \&$ source $=$ web\&cd $=$ 1\&ved=0ahUKEwiPlZqfhqzJAhUO.

Leal, M., y Arias, J. (2009). Enciclomedia: diagnóstico y propuesta de mejora desde las necesidades de los actores. Ponencia presentada en el X Congreso Nacional de Investigación Educativa, México. Recuperado de: http:// www.comie.org.mx/congreso/memoriaelectronica/ v10/pdf/area_tematica_07/ponencias/0572-F.pdf.

León, W., y Gómez, M. (2007). Usos matemáticos de Internet para la enseñanza secundaria. Una investigación sobre WebQuests de Geometría. Revista Iberoamericana de Educación Matemática, (9), 17-34. Recuperado de: http://www.fisem.org/web/union/revistas/9/ Union_009_007.pdf.

Lerner, D. (2012). La incorporación de las TIC en el aula. Un desafío para las prácticas escolares de lectura y escritura en las TIC en la escuela. En D. Goldin, M. Kriscautzky y F. Perelman (eds.), Las TIC en la escuela, nuevas herramientas para viejos y nuevos problemas (pp. 2388). México: Océano Travesía.

Melhuish, E. C., Sylva, K., Sammons, P., Siraj-Blatchford, I., Taggart, B., y Phan, M. (2008). Effects of the home learning environment and preschool center experience upon literacy and numeracy development in early primary school. Journal of Social Issues, 64(1), 95-114.

Miranda, B., Ruíz, A., Vega, R., y Miranda, B. (2017). Alfabetización inicial, competencia lectora y cortometraje: una propuesta de innovación para estudiantes de preescolar y primaria. Revista Electrónica de Investigación Educativa Sonorense, 9(22), 26-40.

Molinari, M., y Corral, E. (2008). La escritura en la alfabetización inicial: producir en grupos en la escuela y el jardín. En Memoria Académica. La Plata: DGCyE. Recuperado de: http://www.memoria.fahce.unlp.edu. ar/libros/pm.1078/pm.1078.pdf.

Molinari, C., y Ferreiro, E. (2007). Identidades y diferencias en las primeras etapas del proceso de alfabetización. Escrituras realizadas en papel y en computadora. Revista Latinoamericana de Lectura, 28(4), 18-30. Recuperado de: http://www.lecturayvida.fahce.unlp. edu. ar/numeros/a28n4/28_04_Molinari.pdf.

NICHD [National Institute for Child Health and Development]-Early Childcare Research Network (2005). Early child care and children's development in the primary grades: Follow up results from the NICHD study of early child care. American Educational Research Journal, 42(3), 537-570.

Orellana, P., y Melo, C. (2014). Ambiente letrado y estrategias didácticas en la educación preescolar chilena. Magis, Revista Internacional de Investigación en Educación, 6(13), 113-128. 
Pruzzo, V. (2005). Tecnologías de la comunicación en la alfabetización inicial: Proyecto A.L.A.S. “T”. Anuario $N^{o}$ 7, 185-197. Recuperado de: http://www.biblioteca. unlpam.edu.ar/pubpdf/anuario_fch/n07a15pruzzo. pdf.

Quinteros, G. (1997). El uso y función de las letras en el periodo prealfabético (Tesis de Maestría). Departamento de Investigaciones Educativas, Centro de Investigación y de Estudios Avanzados del Instituto Pedagógico Nacional (DIE-CINVESTAV-IPN), México. Recuperado de: http:/ /docplayer.es/12278770-El-uso-y-funciondelas-letras-en-el-periodo-pre-alfabetico.html.

Raphael, P. (2011). La fábrica del lenguaje, S.A. Barcelona: Anagrama.

Rolla, A., y Rivadeneira, M. (2006). ¿Por qué es importante y cómo es una educación preescolar de calidad? Revista En Foco. Recuperado de hppt://www.expansiva.cl/ media/en_foco/documentos/19062006104123.pdf.

Sarmiento, W., y Luna, K. (2017). Aplicación del software GeoGebra en prácticas matemáticas bajo una metodología constructivista. Revista Killkana Sociales, 1(2), 45-50.

SEP [Secretaría de Educación Pública] (2017). Aprendiza jes clave para la educación integral. Educación preescolar. Plan $y$ programas de estudio, orientaciones didácticas y sugerencias de evaluación. Recuperado de: https://www.planyprogramasdestudio.sep.gob.mx/descargables/biblioteca/ preescolar/1LpM-Preescolar-DIGITAL.pdf.

SEP (2020). Programa sectorial para la educación 2020-2024.

Recuperado de: https://www.gob.mx/cms/uploads/ attachment/file/562380/Programa_Sectorial_de_ Educaci_n_2020-2024.pdf.

Snow, C., Burns, M., y Griffin, P. (1998). Preventing reading diffculties in young children. Washington: National Academy Press.

Spiro, R., Feltovich, P., Jacobson, M., y Coulson, R. (1991). Cognitive flexibility, constructivism, and hypertext: random access instruction for advanced knowledge acquisition in ill-structured domains. Educational Technology, (31), 24-33.

Teruggi, L., y Molinari, C. (2015). Analisi comparativa del fenomeno dell'alternanza grafo-fonica nella scrittura manoscritta e digitale di bambini di lingua spagnola e italiana di 4 e 5 anni. Rassegna di Psicologia, 32(2), 11-29. Recuperado de: http://www.memoria.fahce.unlp.edu. ar/art_revistas/pr.7255/pr.7255.pdf.

Vernon, S. (1991). El proceso de construcción de la correspondencia sonora en la escritura (en la transición entre los periodos presilábicos y el silábico) [Tesis de Maestría]. Departamento de Investigaciones Educativas, Centro de Investigación y de Estudios Avanzados del Instituto Pedagógico Nacional (DIE-Cinvestav-IPN), México.

Vernon, S. (1997). La relación entre la conciencia fonológica y los niveles de conceptualización de la escritura [Tesis de Doctorado]. Departamento de Investigaciones Educativas, Centro de Investigación y de Estudios Avanzados del Instituto Pedagógico Nacional (DIE-CinvestavIPN), México.

Vernon, S. (2004). ¿Qué tanto es un pedacito? El análisis que los niños hacen de las partes de la palabra. En A. Pellicer y S. Vernon (eds.), Aprender y enseñar la lengua escrita en el aula (19-40). México: Ediciones MS.

Vernon, S., y Alvarado, M. (2001). Actividades didácticas para la enseñanza de la lectura y la escritura al inicio de la alfabetización. Revista Psicología y Sociedad, (esp.), 51-78.

Vidales, I. (2007). El programa Enciclomedia en escuelas primarias de Nuevo León. En Memoria electrónica del IX Congreso Nacional de Investigación Educativa del COMIE, México.

Williner, B. (2011). Estudio de habilidades matemáticas cuando se realiza actividades usando software específico. Revista Iberoamericana de Educación Matemática, (27), 115-129.

Cómo citar este artículo:

Calderón Guerrero, G., y Pohls Díaz, E. (2021). La influencia de un recurso informático en el proceso de alfabetización inicial: Un estudio con niños de tercero de preescolar. IE Revista de Investigación Educativa de la REDIECH, 12, e1176.doi: 10.33010/ie_rie_rediech.v12i0.1176. 